\title{
Expression of TWEAK/Fn14 in neuroblastoma: Implications in tumorigenesis
}

\author{
INGVILD PETTERSEN $^{1}$, NINIB BARYAWNO ${ }^{2}$, FRIDA ABEL $^{3}$, WENCHE HELEN BAKKELUND ${ }^{4}$, \\ SVETLANA N. ZYKOVA ${ }^{4}$, JAN-OLOF WINBERG ${ }^{5}$, UGO MOENS $^{6}$, AGNES RASMUSON $^{2}$, \\ PER KOGNER $^{2}$, JOHN INGE JOHNSEN ${ }^{2}$ and BALDUR SVEINBJÖRNSSON ${ }^{2,6}$ \\ ${ }^{1}$ Translational Cancer Research Group, Institute of Clinical Medicine, University of Troms $\varnothing$, Troms $\varnothing$, Norway; \\ ${ }^{2}$ Childhood Cancer Research Unit, Department of Women and Children's Health, Karolinska Institutet, \\ Stockholm; ${ }^{3}$ Department of Clinical Genetics, The Sahlgrenska Academy at Gothenburg University, \\ Gothenburg, Sweden; Departments of ${ }^{4}$ Cell Biology and Histology and ${ }^{5}$ Medical Biochemistry, \\ ${ }^{6}$ Research Group of Molecular Inflammation, Institute of Medical Biology, \\ University of Troms $\emptyset$, Troms $\emptyset$, Norway
}

Received October 22, 2012; Accepted November 30, 2012

DOI: $10.3892 /$ ijo.2013.1800

\begin{abstract}
Tumor necrosis factor-like weak inducer of apoptosis (TWEAK), a member of the tumor necrosis factor (TNF) family of cytokines, acts on responsive cells via binding to a cell surface receptor called Fn14. TWEAK binding to an Fn14 receptor or constitutive Fn14 overexpression has been shown to activate nuclear factor $\kappa \mathrm{B}$ signaling which is important in tumorigenesis and cancer therapy resistance. In the present study, we demonstrate that TWEAK and Fn14 are expressed in neuroblastoma cell lines and primary tumors, and both are observed at increased levels in high-stage tumors. The treatment of neuroblastoma cell lines with recombinant TWEAK in vitro causes increased survival, and this effect is partially due to the activation of $\mathrm{NF}-\kappa \mathrm{B}$ signaling. Moreover, TWEAK induces the release of matrix metalloprotease-9 (MMP-9) in neuroblastoma cells, suggesting that TWEAK may play a role in the invasive phase of neuroblastoma tumorigenesis. TWEAK-induced cell survival was significantly reduced by silencing the TWEAK and Fn14 gene functions by siRNA. Thus, the expression of TWEAK and Fn14 in neuroblastoma suggests that TWEAK functions as an important regulator of primary neuroblastoma growth, invasion and survival and that the therapeutic intervention of the TWEAK/Fn14 pathway may be an important clinical strategy in neuroblastoma therapy.
\end{abstract}

Correspondence to: Professor Baldur Sveinbjörnsson, Research Group of Molecular Inflammation, Institute of Medical Biology, University of Troms $\varnothing$, Troms $\emptyset$, Norway

E-mail: baldur.sveinbjornsson@uit.no

Key words: neuroblastoma, tumor necrosis factor-like weak inducer of apoptosis, Fn14, nuclear factor $\kappa \mathrm{B}$

\section{Introduction}

The tumor necrosis factor (TNF) superfamily consists of numerous cytokine ligands and receptors that regulate many biological processes, including cell proliferation and survival, and their balance is important in maintaining normal cellular functions $(1,2)$.

TNF superfamily ligands are mostly expressed as type II transmembrane proteins that can be processed into smaller soluble proteins exerting a wide variety of biological effects by binding their cognate family of TNF receptors $(1,2)$. Tumor necrosis factor-like weak inducer of apoptosis (TWEAK), a member of the tumor necrosis factor superfamily, was first described as an inducer of apoptosis in transformed cell lines (3). It has later become apparent that TWEAK is a multifunctional cytokine that regulates survival (4), proliferation (5-7), migration $(6,8,9)$, differentiation $(10,11)$, and induces apoptosis in certain tumor cell lines $(12,13)$. TWEAK acts on responsive cells via binding to an inducible high affinity cell surface receptor known as fibroblast growth factor-inducible 14 (Fn14) (14). It has been demonstrated that TWEAK binding to Fn14, or constitutive Fn14 overexpression, activates nuclear factor $\kappa \mathrm{B}$ $(\mathrm{NF}-\kappa \mathrm{B})$ signaling pathway, which is known to play a pivotal role in immune- and inflammatory processes, oncogenesis and cancer therapy resistance $(15,16)$.

The aberrant expression of both TWEAK and Fn14 has been detected in human tumor samples $(7,17)$, and the possibility that TWEAK and Fn14 could contribute to tumor growth has recently been reviewed $(16,18,19)$. TWEAK activates cellular processes attributed to tumor growth such as matrix metalloprotease (MMP) secretion (20-22), migration $(6,8,9)$, proliferation (5-7), and apoptotic resistance (4). TWEAK is also mitogenic for endothelial cells and stimulates angiogenesis (5,23).

Neuroblastoma, an embryonic tumor of the sympathetic nervous system, is the most common and deadly extracranial tumor associated with childhood. Neuroblastoma 
exhibits heterogeneous biological and clinical features ranging from spontaneous regression to highly malignant disease with metastatic spread. More than $40 \%$ of children with neuroblastoma are diagnosed as high-risk patients and despite intensive treatment modalities; the cure rate for these patients is less than $50 \%$ (24). Hence, there is a great need for new therapies based on a biological understanding of this disease.

The aim of this study was to assess the expression of TWEAK and Fn14 in childhood neuroblastoma, particularly how it relates to the functional importance in neuroblastoma cell growth and survival.

\section{Materials and methods}

Reagents and antibodies. Phospho NF-kB p65 (Ser536; cat. 3031), 65 kDa, NF- $\mathrm{kB}$ p65 (cat. 3034) 65 kDa and Fn14 polyclonal antibody were purchased from Cell Signaling (Beverly, MA, USA). NF- $\kappa$ B p65 (sc-109), TWEAK (FL-249), and TWEAK (S-20) antibodies were purchased from Santa Cruz Biotechnology (Santa Cruz, CA, USA). Secondary anti-goat Alexa 594 and anti-rabbit Alexa 488 antibodies were purchased from Invitrogen (Carlsbad, CA, USA). Human recombinant IL- $1 \beta$, TNF- $\alpha$ and TWEAK were purchased from R\&D Systems (Abingdon, UK), and human TWEAK ELISA was purchased from PeproTech (London, UK).

Human tissue samples. Primary neuroblastoma samples from tumors and non-malignant adrenals were obtained during surgery, snap-frozen in liquid nitrogen, and transferred to $-80^{\circ} \mathrm{C}$ for future analysis. Twenty six neuroblastoma samples derived from children of different ages and all clinical stages, including different biological subsets (MYCN amplification, 7 of 27; 1p deletion, 9 of 27; Table I) were analyzed. Three childhood ganglioneuromas and three samples of non-malignant adrenals from children aged 12-25 months were also included. Ethical approval was obtained from the Karolinska University Hospital Research Ethics Committee.

Neuroblastoma cell lines. Human neuroblastoma cell lines [SK-N-BE(2), SK-N-DZ, SH-SY5Y, SK-N-SH, SK-N-FI, IMR-32, SK-N-AS and SHEP-1] were grown in RPMI-1640 medium supplemented with $10 \%$ fetal calf serum (FCS), $2 \mathrm{mM}$ L-glutamine, and antibiotics. The cultures were kept at $37^{\circ} \mathrm{C}$ in a humified $5 \% \mathrm{CO}_{2}$ atmosphere.

$R N A$ isolation and RT-PCR. Total RNA was extracted from cultured neuroblastoma cells using RNeasy Mini kit (cat. no. 74104, Qiagen, Valencia, CA, USA) according to the manufacturer's instructions. cDNA was synthesized using $2.0 \mu \mathrm{g}$ total RNA that was reverse transcribed in a final volume of $50 \mu 1$ using the SuperScript preamplification kit (Life Technologies, Inc., Gaithersburg, MD, USA).

Gene specific PCR was performed in $50 \mu 1$ of reaction mixture containing 2-10 $\mu \mathrm{l}$ cDNA (from isolated RNA), $2.5 \mathrm{U}$ of Taq DNA polymerase (Promega, Madison, WI, USA), $10 \mathrm{mM}$ Tris- $\mathrm{HCl}(\mathrm{pH} 8.0), 100 \mathrm{mM} \mathrm{KCl}, 0.1 \%$ Triton ${ }^{\circledR} \mathrm{X}-100$, $2.0 \mathrm{mM} \mathrm{MgCl}_{2}, 1 \mathrm{mM}$ deoxynucleotide triphosphate mix, and $1 \mu \mathrm{M}$ of each primer.
PCR for TWEAK and Fn14 was performed as follows: $94^{\circ} \mathrm{C}$ for $5 \mathrm{~min}$ (first denaturation/hot start) and then at $94^{\circ} \mathrm{C}$ for 1 (denaturation), $52^{\circ} \mathrm{C}$ for $1.5 \mathrm{~min}$ (annealing), and $72^{\circ} \mathrm{C}$ for $1 \mathrm{~min}$ (extension) for 35 cycles with a $10 \mathrm{~min}$ final extension at $72^{\circ} \mathrm{C}$. PCR conditions for $\beta$-actin were identical except for a $1 \mathrm{~min}$ annealing at $55^{\circ} \mathrm{C}$, and a total of 27 cycles, with a $10 \mathrm{~min}$ final extension.

PCR amplifications were performed in a PTC-200 Peltier Thermal Cycler (MJ Research Inc. Waltham, MA, USA). PCR products were analyzed by agarose gel $(1.5 \%)$ electrophoresis and photographed under UV light. Nucleotide sequences of PCR primers used were as follows: Fn14, 5'-GAC CTG GAC AAG TGC ATG GAC-3' (sense) and 5'-AGC TGT TTT GTG TGA GCC AGC- 3' (antisense); TWEAK 5'-ATC GCA GCC CAT TAT GAA GTT C-3' (sense) and 5'-GAT GGA AAA CAC GTG AAC AGG C-3' (antisense); $\beta$-actin, 5'-TGA CGG GGT CAC CCA CAC TGT GCC CAT CTA-3' (sense) and 5'-ACT CGT CAT ACT CCT GCT TGC TGA TCC A-3' (antisense); PCR fragments of 500 (Fn14), 607 (TWEAK), and 625 bp $(\beta$-actin) were expected.

Microarray expression analysis. Raw data files from four European expression microarray studies generated from two Affymetrix platforms (HU133A and HU133plus2) were obtained from ArrayExpress (www.ebi.ac.uk/microarray-as/ ae/) and the r2 data base (http://hgserver1.amc.nl/cgi-bin/r2/ main.cgi). The three studies run on the HU133A platform (25-27), were reanalyzed using gcRMA by Bioconducter for R 2.9.2 (library BioC 2.4) in two separate groups: i) De Preter data set comprising preamplified primary neuroblastoma samples ( $n=17$, stages 1-4), ii) McArdle $(n=16)$ and Wilzén ( $\mathrm{n}=8$ ) data sets comprising non-preamplified primary neuroblastoma samples. Also, expression values $(\log 2)$ from 76 MAS5.0 normalized neuroblastoma samples (stages 1-4) run on the Affymetrix HU133plus2 platform were obtained from the $\mathrm{r} 2$ database (28) and referred to as the Versteeg data set.

Neuroblastoma samples from all three data sets (25-27) were divided into two groups based on their clinical stage (INSS stage) (29) and investigated for differential expression of TNFSF12 (TWEAK) and TNFRSF12A (Fn14) between groups. The significance was tested by Welch's t-test (2-tailed, 2 sample comparison, unequal variance).

Treatment of cells with TWEAK. To prevent influence of endogenously produced TWEAK, neuroblastoma cells were serum starved in an RPMI-1640 medium containing $0.1 \%$ FCS for $24 \mathrm{~h}$ prior to the incubation of recombinant human TWEAK for the indicated concentrations and timepoints.

Immunohistochemistry. Formalin-fixed and paraffin-embedded tissue sections were deparaffinized in xylene and graded alcohols, hydrated and washed in PBS. After antigen retrieval in sodium citrate buffer ( $\mathrm{pH}$ 6.0) in a microwave oven, the endogenous peroxidase was blocked by $0.3 \% \mathrm{H}_{2} \mathrm{O}_{2}$ for $15 \mathrm{~min}$. Sections were incubated overnight at $4^{\circ} \mathrm{C}$ with primary antibody (TWEAK; FL-249). As a secondary antibody, the antirabbit horseradish peroxidase (HRP) SuperPicTure Polymer detection kit was used (Zymed-Invitrogen, San Francisco, CA, 
Table I. Immunohistochemical assessment of TWEAK, Fn14 and pNF- $\kappa$ B expression in neuroblastoma tissue samples.

\begin{tabular}{|c|c|c|c|c|c|c|c|c|c|c|c|c|}
\hline Sample & DIA $^{a}$ & $\begin{array}{l}\text { Age } \\
\text { months }\end{array}$ & Gender & $\begin{array}{l}\text { Stage } \\
\text { INSS }^{\text {b }}\end{array}$ & $\begin{array}{c}\text { MYCN } \\
\text { ampl }\end{array}$ & $1 \mathrm{p}$ del & $\begin{array}{l}\text { DNA } \\
\text { ploidy }\end{array}$ & High-risk $^{\mathrm{c}}$ & Outcome & TWEAK & $\begin{array}{l}\text { TWEAK-R/ } \\
\text { Fn-14 }\end{array}$ & pNF- $\kappa \mathrm{B}$ \\
\hline 1 & $\mathrm{NB}^{\mathrm{d}}$ & 21 & M & 1 & No & No & $4 n$ & No & $\mathrm{NED}^{\mathrm{h}}$ & +++ & ++ & +++ \\
\hline 2 & NB & 123 & $\mathrm{~F}$ & 1 & No & No & $3 n$ & No & NED & $++(+)$ & + & +++ \\
\hline 3 & NB & 7 & F & 1 & Yes & Yes & $2 n$ & No & $\mathrm{DOD}^{\mathrm{i}}$ & ++ & ++ & ++ \\
\hline 4 & NB & 13 & M & 1 & No & No & & No & NED & ++ & ++ & ++ \\
\hline 5 & NB & 18 & F & 1 & No & No & & No & NED & ++ & + & +++ \\
\hline 6 & NB & 31 & M & $2 \mathrm{~B}$ & No & No & $3 n$ & No & NED & +++ & ++ & +++ \\
\hline 7 & NB & 33 & $\mathrm{~F}$ & $2 \mathrm{~A}$ & No & No & $3 n$ & No & NED & ++ & + & +++ \\
\hline 8 & NB & 8 & $\mathrm{~F}$ & 2 & No & No & $3 n$ & No & NED & ++ & ++ & +++ \\
\hline 9 & NB & 110 & M & 2 & No & No & $2 n$ & No & NED & +++ & ++ & +++ \\
\hline 10 & NB & 5 & $\mathrm{~F}$ & 2 & No & No & $3 n$ & No & $\mathrm{AWD}^{\mathrm{j}}$ & ++ & + & ++ \\
\hline 11 & NB & 103 & $\mathrm{~F}$ & $2 \mathrm{~B}$ & No & No & $2 n$ & No & NED & +++ & ++ & +++ \\
\hline 12 & NB & 6 & M & 3 & No & ND & $3 n$ & No & NED & +++ & +++ & +++ \\
\hline 13 & NB & 12 & $\mathrm{~F}$ & 3 & No & No & $5 n$ & No & NED & +++ & ++ & ++ \\
\hline 14 & NB & 0 & M & 3 & No & No & $3 n$ & No & $\mathrm{DOC}^{\mathrm{k}}$ & ++ & ++ & +++ \\
\hline 15 & NB & 79 & $\mathrm{M}$ & 3 & Yes & Yes & $3 n$ & Yes & NED & +++ & ++ & +++ \\
\hline 16 & NB & 136 & M & 4 & Yes & Yes & $2 n$ & Yes & DOD & ++ & +++ & +++ \\
\hline 17 & NB & 39 & F & 4 & Yes & Yes & $2 n$ & Yes & DOD & +++ & +++ & +++ \\
\hline 18 & NB & 35 & F & 4 & No & Yes & & Yes & NED & ++ & $++(+)$ & +++ \\
\hline 19 & NB & 28 & M & 4 & Yes & Yes & $3 n$ & Yes & NED & ++ & ++ & +++ \\
\hline $20 \mathrm{a}$ & NB & 8 & M & $4_{M}^{g}$ & No & No & $3 n$ & No & NED & +++ & ++ & +++ \\
\hline $20 \mathrm{~b}$ & NB & 8 & M & $4_{M}$ & No & No & $4 n / 5 n$ & No & NED & +++ & +++ & +++ \\
\hline 21 & NB & 22 & M & 4 & Yes & Yes & & Yes & DOD & +++ & ++ & ++ \\
\hline 22 & NB & 50 & $\mathrm{~F}$ & 4 & Yes & Yes & & Yes & DOD & ++ & ++ & ++ \\
\hline 23 & NB & 0 & M & $4 \mathrm{~S}$ & No & No & $3 n$ & No & NED & +++ & ++ & ++ \\
\hline 24 & NB & 10 & M & $4 \mathrm{~S}$ & No & No & $3 n$ & No & NED & +++ & ++ & ++ \\
\hline 25 & NB & 0 & M & $4 S$ & No & ND & $4 n$ & No & NED & +++ & ++ & +++ \\
\hline
\end{tabular}

Ampl, amplification. Del, deletion. +, low. ++, median. +++, significantly strong.

USA). A matched isotype control was used as a control for non-specific background staining. Routine standard staining showing a normal histology of neuroblastoma was performed with hematoxylin and eosin.

For immunofluorescence studies, cells were grown on fibronectin-coated chamber slides (Nunc, Roskilde, Denmark) for $24 \mathrm{~h}$. Cultures were then washed and fixed with $2 \%$ paraformaldehyde for $15 \mathrm{~min}$ and $70 \%$ cold methanol for $5 \mathrm{~min}$. After washing with PBS buffer, goat-anti TWEAK (S-20) and rabbit-anti Fn14 antibodies were incubated with cultures overnight at $4^{\circ} \mathrm{C}$. After rinsing in PBS, cultures were incubated with secondary antibodies conjugated with Alexa 488 and Alexa 599, respectively. A matched isotype control was used as a control for non-specific background staining. The cells were examined in a Zeiss axiophot photomicroscope (Carl Zeiss, Oberkochen, Germany). Nuclear translocation of NF- $\kappa$ B upon stimulation with TWEAK was performed by immunofluorescence studies using anti-NF- $\kappa$ B p 65 antibody (sc-109) that recognizes both non-phosphorylated and phosphorylated forms of NF- $\mathrm{NB}$ p65.
TWEAK ELISA. TWEAK ELISA was performed to measure the endogenous production of TWEAK in neuroblastoma cells upon stimulation by cytokines IL-1 $\beta$ and TNF- $\alpha$. Neuroblastoma cells [SK-N-AS, SK-N-BE(2) and SH-SY5Y] were seeded in a regular growth medium in 96-well plates and allowed to attach. Cells were then treated with two concentrations (10 and $50 \mathrm{ng} / \mathrm{ml}$ ) of IL-1 $\beta$ and TNF- $\alpha$, respectively, for $12 \mathrm{~h}$. Supernatants from treated cells were collected and $100 \mu 1$ medium per well were analyzed by using the Human TWEAK ELISA Development kit (PeproTech, Rocky Hill, NJ, USA), following the manufacturer's instructions.

Protein isolation and immunoblotting. Proteins from TWEAK-treated cells and control cells were extracted in RIPA lysis buffer (cat. no. 20-188, Upstate Biotechnology, USA) containing complete, mini, EDTA-free protease inhibitor (cat. no. 11836170 001, Roche). The protein content was measured using Bradford reagents (Bio-Rad Laboratories, CA, USA). Equal amounts of protein were separated by NuPAGE, Novex 
and Tris-Acetat Mini Gels (Invitrogen) 4-12\% in reduced conditions, and proteins were transferred to a PVDF (Pierce, Rockford, IL, USA) membrane and incubated with primary antibodies at $4^{\circ} \mathrm{C}$ overnight. Alkaline-phosphatase conjugated antibodies were used as secondary antibodies. Detection and visualization were performed using Pierce Super Signaling solutions (Pierce), the Fujifilm Luminescent Image Analyzer LAS-3000 and the Fujifilm MultiGauge (Ver. 3.0) analysis software.

$N F-\kappa B$ luciferase reporter gene assay. The NF- $\kappa \mathrm{B}-$ responsive reporter plasmid $\kappa$ Bcon A-LUC was provided by E. Sontag (30) and also described in Johannesen et al (31). SK-N-AS and SK-N-BE cells were seeded in 6-well plates in triplicates the day before transfection. Cells were transfected using Lipofectamine 2000 (Invitrogen), $4 \mu \mathrm{g} / \mu \mathrm{l}$ DNA per well and calf thymus DNA (Amersham Pharmacia, Sweden), and incubated for $6 \mathrm{~h}$ at $37^{\circ} \mathrm{C}$ in a humidified $5 \% \mathrm{CO}_{2}$ atmosphere. Cells were then serum starved for $14 \mathrm{~h}$ and subsequently incubated for $6 \mathrm{~h}$ with TWEAK $(100 \mathrm{ng} / \mathrm{ml})$ or TNF- $\alpha(30 \mathrm{ng} / \mathrm{ml})$. Cells were washed in 1X PBS and lysed in TROPIX lysis buffer containing $0.5 \mathrm{mM}$ DTT. Luciferase activity was determined using the Dual-Light Luciferase Gene Assay System (Applied Biosystems Inc., Foster City, CA, USA) in a Luminoscan RT (Labsystems, Helsinki, Finland). Luciferase measurements were corrected to protein concentrations in each cell lysate, and protein measurements were performed as described above. The difference between the groups was analyzed on log-transformed values after normalization to total protein content in the cell lysates using a repeated measures one-way ANOVA test, followed by a Bonferroni multiple comparison test.

Fn14 and TWEAK siRNA. SK-N-AS and SK-N-BE(2) cells were seeded in 6-well culture plates in RPMI medium at a $30-50 \%$ confluence. Cells were transfected with target-specific Fn14 (sc-43764), TWEAK (sc-37522), control (Fluorescein conjugate-A) (sc-36869) or scrambled control (sc-37007) siRNA (Santa Cruz Biotechnology), respectively, at a concentration of $33 \mathrm{nM}$ using Lipofectamine 2000 in OptiMEM. To evaluate cell viability, western blot analysis of protein extracts and trypan blue exclusion assay were performed $72 \mathrm{~h}$ after the initial transfection. Transfection efficiency was assessed in SK-N-AS cells transfected with control (Fluorescein conjugate-A) using flow cytometry.

Detection of MMP-2 and MMP-9 in cell conditioned media using gelatin zymography. The presence of MMP-2 and MMP-9 in serum-free media from cells treated with or without recombinant TWEAK was determined by SDS-gelatin zymography. Approximately 30,000 cells were seeded in 96-well plates and left to attach in 10\% RPMI-1640 overnight. Then, SK-N-AS and SK-N-SH cells were starved in 0.1\% RPMI-1640 for $24 \mathrm{~h}$ prior to incubation with TWEAK (0-1000 $\mathrm{ng} / \mathrm{ml})$ for $48 \mathrm{~h}$. SDS-substrate PAGE was done as previously described $(32,33)$ with gels containing $0.1 \%(\mathrm{w} / \mathrm{v})$ gelatin. The gelatin zymograms were calibrated with a mixture of conditioned serum-free medium from THP-1 and humans skin fibroblast cells (32). Conditioned medium $(10 \mu \mathrm{l})$ was mixed with $2.5 \mu \mathrm{l}$ of loading buffer (250 mM Tris- $\mathrm{HCl}, \mathrm{pH} 6.8,10 \%$ SDS,
$0.03 \%$ bromophenol blue and $50 \%$ glycerol). Eight $\mu 1$ of this non-heated mixture was applied to the gel, which was run at $20 \mathrm{~mA} /$ gel at $4^{\circ} \mathrm{C}$. Thereafter, the gel was washed twice in $100 \mathrm{ml}$ of washing buffer [2.5\% (v/v) Triton X-100 in water], and then incubated in $100 \mathrm{ml}$ of assay buffer $(50 \mathrm{mM}$ Tris- $\mathrm{HCl}$, $\mathrm{pH} 7.5,5 \mathrm{mM} \mathrm{CaCl}_{2}, 0.2 \mathrm{M} \mathrm{NaCl}$ and $0.02 \%$ Brij-35) for $20 \mathrm{~h}$ at $37^{\circ} \mathrm{C}$. Gels were stained with $0.2 \%$ Coomassie brilliant blue $\mathrm{R}-250$ (30\% methanol) and destained in a solution containing $30 \%$ methanol and $10 \%$ acetic acid. Gelatinase activity was evident as cleared regions.

Statistical analyses. All statistical analyses were performed with GraphPad Prism Software (GraphPad Software, San Diego, CA, USA). The t-test was used to determine whether the mean of a single sample differed significantly from control. To compare several treatment groups, one-way ANOVA with Tukey multiple-comparisons tests were used. $\mathrm{P}<0.05$ was considered statistically significant.

\section{Results}

TWEAK and Fn14 are expressed in neuroblastoma primary tumors and cell lines. We investigated eight different neuroblastoma cell lines for the expression of TWEAK and Fn14. All human neuroblastoma cell lines investigated showed varying degrees of both TWEAK and Fn14 mRNA and protein expression as detected by RT-PCR and western blot analysis, respectively (Fig. 1A and B). The 30-35-kDa band for TWEAK represents the transmembrane form of TWEAK as the soluble form could not be detected by western blot analysis. We selected 3 cell lines SK-N-AS, SK-N-BE(2) and $\mathrm{SH}-\mathrm{SY} 5 \mathrm{Y}$ for the in vitro experiments. The rationale behind choosing these cell lines was based on the genetical and phenotypical differences between the cell lines. SK-N-AS and SH-SY5Y are typical non-MYCN-amplified cell lines, whereas the SK-N-BE(2) cell line is $M Y C N$-amplified and P53-mutated. Furthermore, while the SK-N-BE(2), SK-N-SH and SK-N-AS show multi-drug resistant (MDR) phenotype, the SH-SY5Y cell line does not. In order to investigate whether neuroblastoma cells were able to produce and secrete TWEAK in vitro, we assessed the TWEAK production by ELISA. The TWEAK levels in cell supernatants from SK-N-AS cells showed an elevated secretion upon stimulation with pro-inflammatory cytokines IL-1 $\beta$ and TNF- $\alpha$ (Fig. 1C). Similar results were obtained for SH-SY5Y and SK-N-BE(2) cell lines (data not shown).

Immunofluorescence staining of neuroblastoma SK-N-AS cells stained with antibodies towards TWEAK (red) and Fn14 (green) revealed the cellular distribution of the ligand and receptor, demonstrating a distribution of TWEAK and Fn14 in the cytoplasm but also to a certain degree to the nuclear compartment (Fig. 1D).

The staining of primary neuroblastoma tumor tissue with antibodies against TWEAK and Fn14 revealed significant cytoplasmic and nuclear expression of both TWEAK and Fn14 in all primary tumors that were analyzed (Fig. 1E and Table I). No significant difference in staining intensity between favorable and non-favorable tumors ( $M Y C N$-amplified vs non- $M Y C N$ amplified) could be detected by immunohistochemistry and no staining was observed in sections incubated with isotype 
A

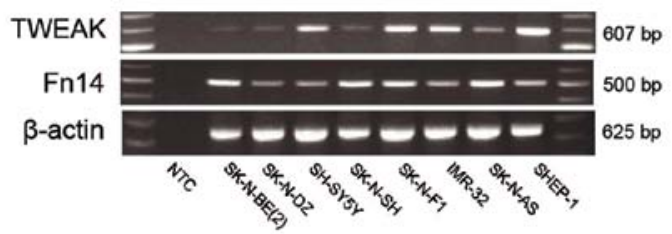

B

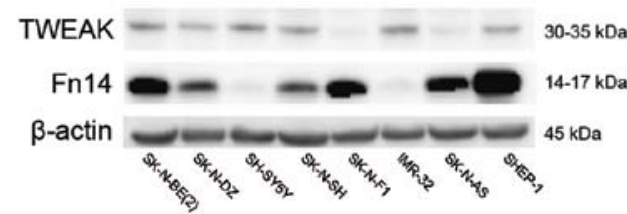

D
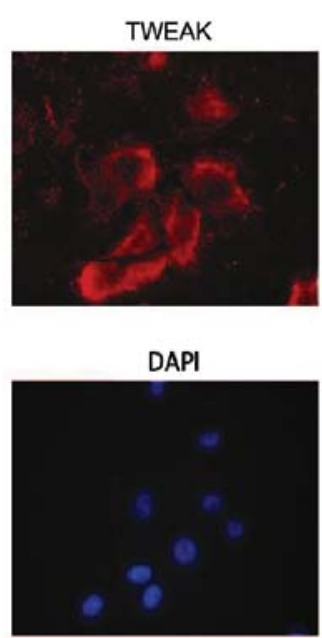
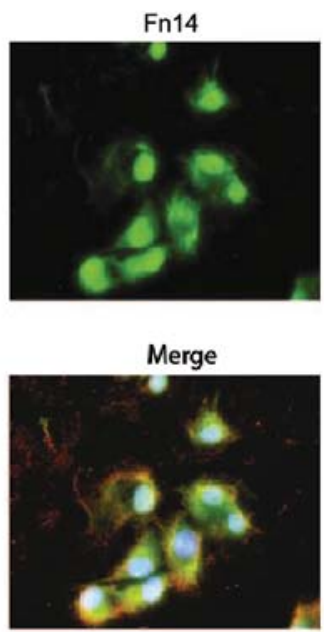

C

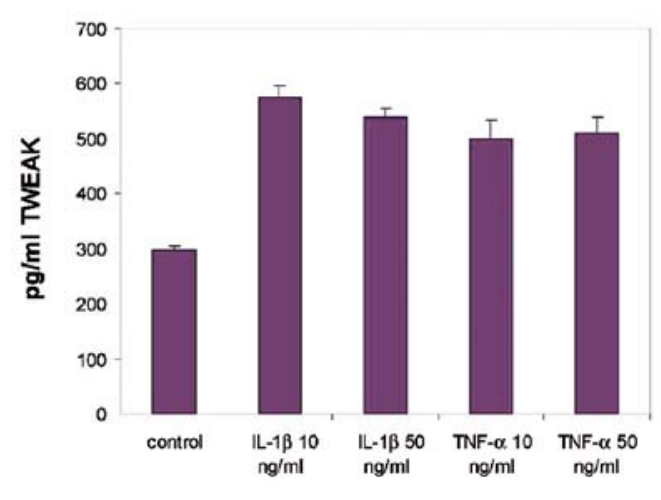

E

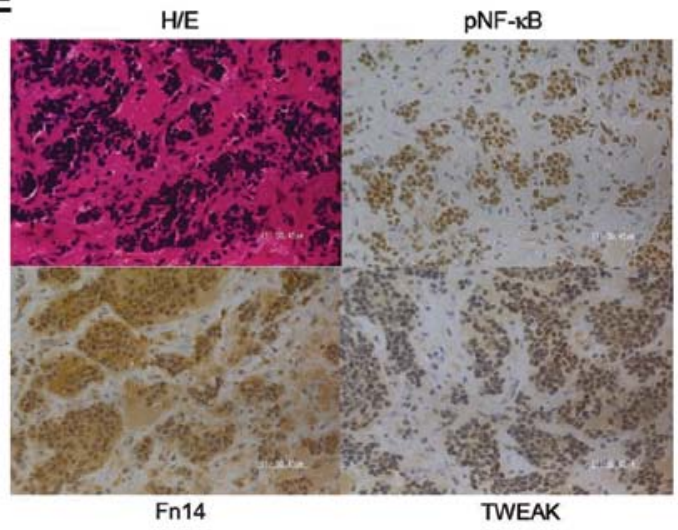

Figure 1. TWEAK and Fn14 expression and secretion in neuroblastoma cell lines and primary tumors. (A) RT-PCR showed expression of TWEAK and Fn14 in all neuroblastoma cell lines investigated. NTC, negative control. (B) Western blot analysis detected protein bands of $\sim 30-35 \mathrm{kDa}$ corresponding to TWEAK and 14-17 kDa corresponding to Fn14 in neuroblastoma cell lines. $\beta$-actin was used to ensure equal loading. (C) TWEAK secretion in neuroblastoma cells. SK-N-AS cells were treated with 10 or $50 \mathrm{ng} / \mathrm{ml} \mathrm{IL-1} \beta$ and TNF- $\alpha$ and cell supernatants were analyzed for TWEAK secretion measured by ELISA. (D) Immunofluorescence images of neuroblastoma cell line SK-N-AS showing cellular distribution of TWEAK (red) and Fn14 (green). Merge shows colocalization of ligand and receptor. DAPI shows staining of the nucleus. (E) Immunohistochemical detection of TWEAK (x400), Fn14 (x200) and phospho-NF- $\mathrm{B}$ (x200) in primary human neuroblastoma tumor tissue (H/E hematoxylin/eosin; $\mathrm{x} 400)$.

control antibody. Moreover, mRNA levels of both TWEAK and its receptor Fn14 were found to be generally higher in primary high stage tumors (stage 3-4) compared to low stage tumors (stage 1-2), when investigating three European microarray data sets (Fig. 2). The up-regulation was significant for TWEAK and Fn14 in one out of three data sets respectively, i.e. the De Preter and Versteeg data sets $(\mathrm{p}<0.05$, Welch's t-test; Fig. 2). Also, the expression variance of both TWEAK and Fn14 was considerably higher in the high-stage group, and the up-regulation seemed to involve a sub-set of tumor cases. Ten out of 117 cases from all three data sets showed up-regulation of TWEAK (fold change >2), 13 showed up-regulation of Fn14 (fold change $>2$ ), and 9 showed up-regulation of both genes (fold change $>2$ ) compared to the mean expression levels of the low-stage group (Fig. 2).

TWEAK promotes $N F-\kappa B$ activation and nuclear translocalization in neuroblastoma cells. TWEAK treatment has previously been shown to stimulate $N F-\kappa B$ activation in different cell types (15,34-36). To determine if TWEAK could induce activation of $\mathrm{NF}-\kappa \mathrm{B}$ in neuroblastoma cells, we examined the intracellular localization of $N F-\kappa B$ in TWEAK stimulated SK-N-AS cells. No nuclear staining for $\mathrm{NF}-\kappa \mathrm{B}$ was observed in untreated cells, but after $20 \mathrm{~min}$ of stimulation with recombinant TWEAK, nuclear translocation of NF- $\kappa \mathrm{B}$ was detected (Fig. 3A). To validate the TWEAK induction of NF- $\kappa \mathrm{B}$ in SK-N-AS cells grown in $0.1 \%$ FCS, we isolated total protein fractions of cells treated with TWEAK $(100 \mathrm{ng} / \mathrm{ml})$ and immunoblotted for both the $\mathrm{NF}-\kappa \mathrm{B}$ and the $\mathrm{pNF}-\kappa \mathrm{B}$ subunit. Blots revealed an increased level of $\mathrm{pNF}-\kappa \mathrm{B}$ (p65) subunit shortly after stimulation by TWEAK at the same time that NF- $\kappa$ B showed a decreased signal (Fig. 3B). This is consistent with the immunostaining results showing activation of $\mathrm{pNF}-\kappa \mathrm{B}$ (Fig. $3 \mathrm{~A}$ ), and the results of SK-N-AS cells upon stimulation with TWEAK showing increase of $N F-\kappa B$ transcriptional activity upon transient transfection using the $\mathrm{NF}-\kappa \mathrm{B}$-responsive reporter plasmid $\kappa$ Bcon A-LUC (Fig. 3C). 
TNFSF12 (TWEAK)

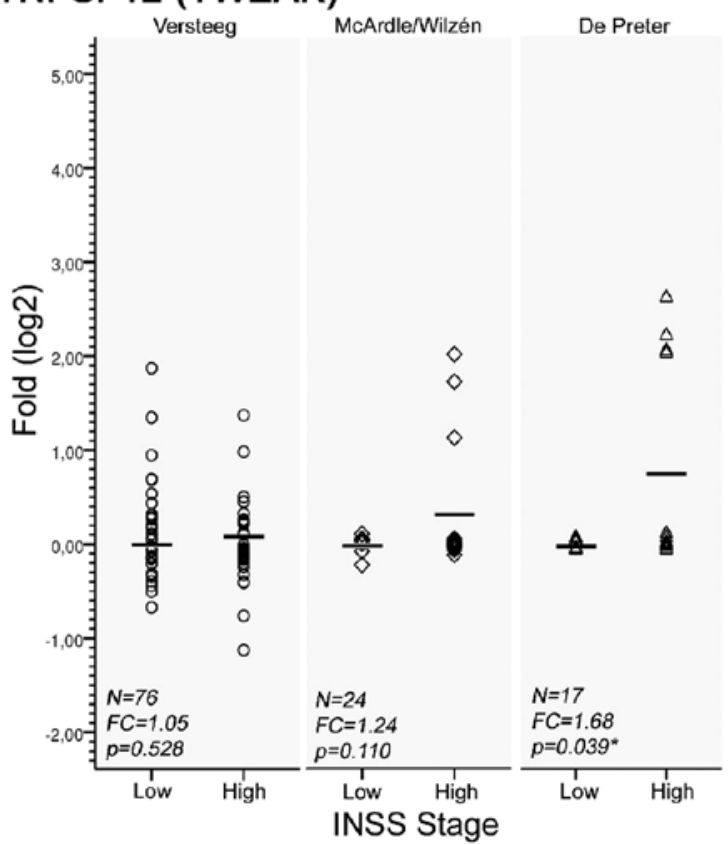

TNFRSF12A (Fn14)

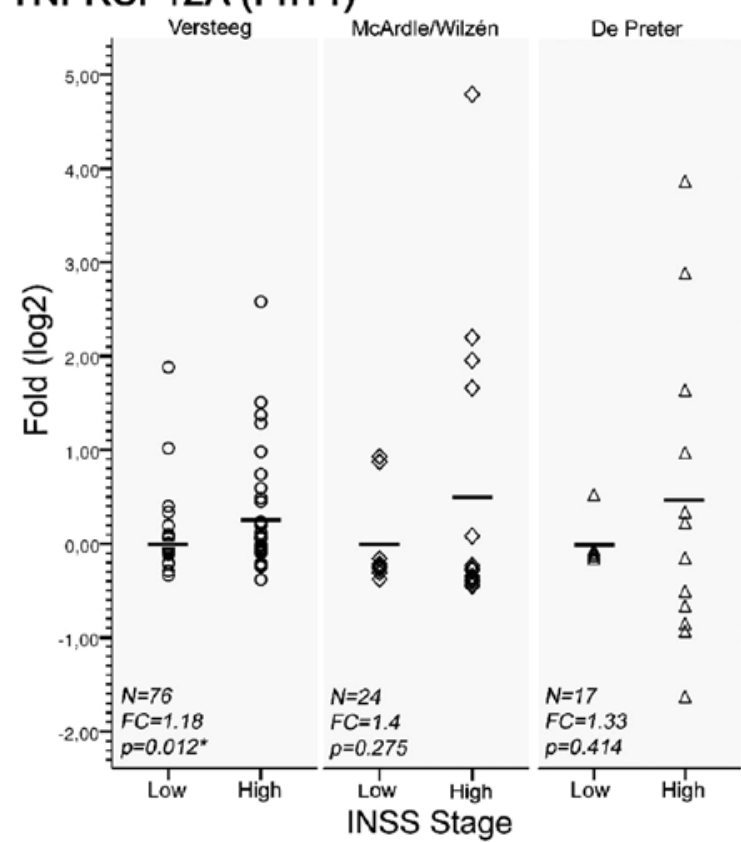

Figure 2. Fold scatter plot of TWEAK (TNFSF12) and Fn14 (TNFRSF12A) mRNA expression in primary tumors. The $\log 2$ fold between high stage (INSS stage 3-4) and low stage (INSS stage 1-2) tumors from four Affymetrix microarray studies, presented as three data sets are plotted. The $\log 2$ fold mean in each group is marked by horizontal line and related to the mean in low stage tumors ( $\log 2$ fold, 0 ). Open circles, Versteeg data set (28) from the $\mathrm{r} 2$ database data set (http://hgserver1.amc.nl/cgi-bin/r2/main.cgi. Open squares, McArdle/Wilzén data set $(26,27)$. Open triangles, De Preter data set (25). N, the number of cases. FC, relative fold change between groups. $\mathrm{p}$, significance by Welch's t-test ( $\mathrm{p}<0.05)$. See text for more detail.

Furthermore, immunohistochemical analysis of neuroblastoma primary tumors using phospho-specific NF- $\mathrm{NB}$ antibody (p65) revealed a significant nuclear staining (Fig. 1E).

Silencing of TWEAK and Fn14 reduces neuroblastoma cell viability. To investigate the influence of TWEAK on neuro-
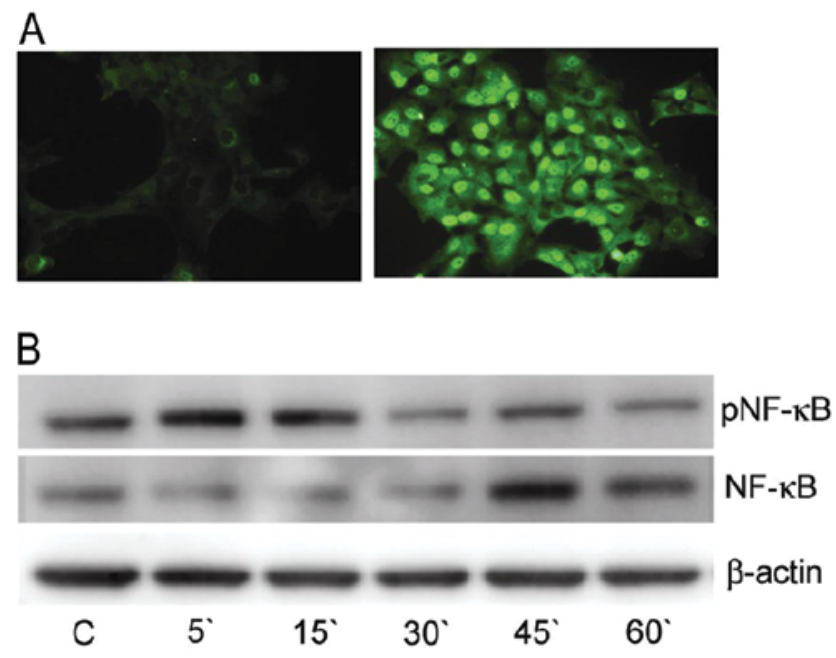

C

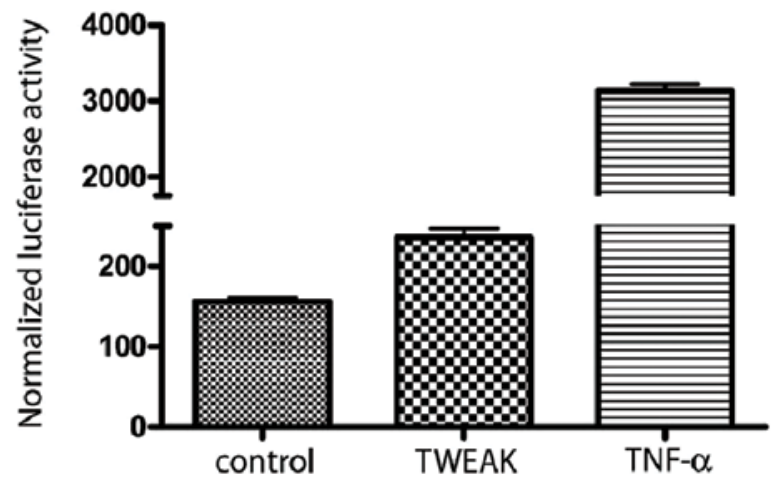

Figure 3. Effect of TWEAK treatment on NF- $\mathrm{B}$ cellular localization in neuroblastoma cells. (A) NF- $\mathrm{NB}$ immunostaining of TWEAK-stimulated neuroblastoma cells. SK-N-AS cells were cultured under reduced serum conditions $(0.1 \%)$ for $16 \mathrm{~h}$ prior to stimulation with TWEAK $(100 \mathrm{ng} / \mathrm{ml})$. Cells were fixed and immunostained for the p65 subunit of NF- $\kappa \mathrm{B}$. The left panel shows non-treated SK-N-AS cells, whereas the right panel shows cells treated with TWEAK for $20 \mathrm{~min}$. (B) TWEAK stimulates NF- $\mathrm{B}$ p65 phosphorylation in neuroblastoma cells. SK-N-AS cells were cultured under reduced serum conditions $(0.1 \%)$ for $16 \mathrm{~h}$ prior to stimulation with TWEAK $(100 \mathrm{ng} / \mathrm{ml})$ for various time periods as indicated or reduced serum for control. Following treatment cells were lysed and total cell extracts were immunoblotted for both the phospho-NF- $\kappa$ B and NF- $\kappa$ B subunit. $\beta$-actin was used as a control for equal loading. (C) TWEAK induces NF- $\kappa \mathrm{B}$ transcriptional activity in neuroblastoma cells. SK-N-AS cells transiently transfected with a NF- $\kappa \mathrm{B}$ plasmid containing luciferase reporter gene were exposed to TWEAK (100 ng/ml), TNF- $\alpha(30 \mathrm{ng} / \mathrm{ml})$ or culture medium only (control) for $6 \mathrm{~h}$. Luciferase activity was measured in cell lysates and values were normalized to total protein content. The experiment was repeated three times with similar results. Data presented are mean \pm SEM from a representative experiment, $\mathrm{n}=3$ per group.

blastoma cell survival, SK-N-AS cells were transfected with siRNA targeting TWEAK or Fn14. As shown in Fig. 4A, the silencing of both TWEAK and Fn14 resulted in significant decrease in neuroblastoma cell survival compared to cells transfected with a scrambled siRNA construct $(\mathrm{p}<0.05)$. Addition of recombinant TWEAK partly restored TWEAK expression and cell survival in TWEAK siRNA-treated SK-N-AS cells, underscoring a role for TWEAK in cell survival (Fig. 4A and B). Increased expression of Fn14 in TWEAK siRNA-treated cells compared to scramble- and nontreated cells (Figs. 1B and 4B) is possibly due to the absence 
A
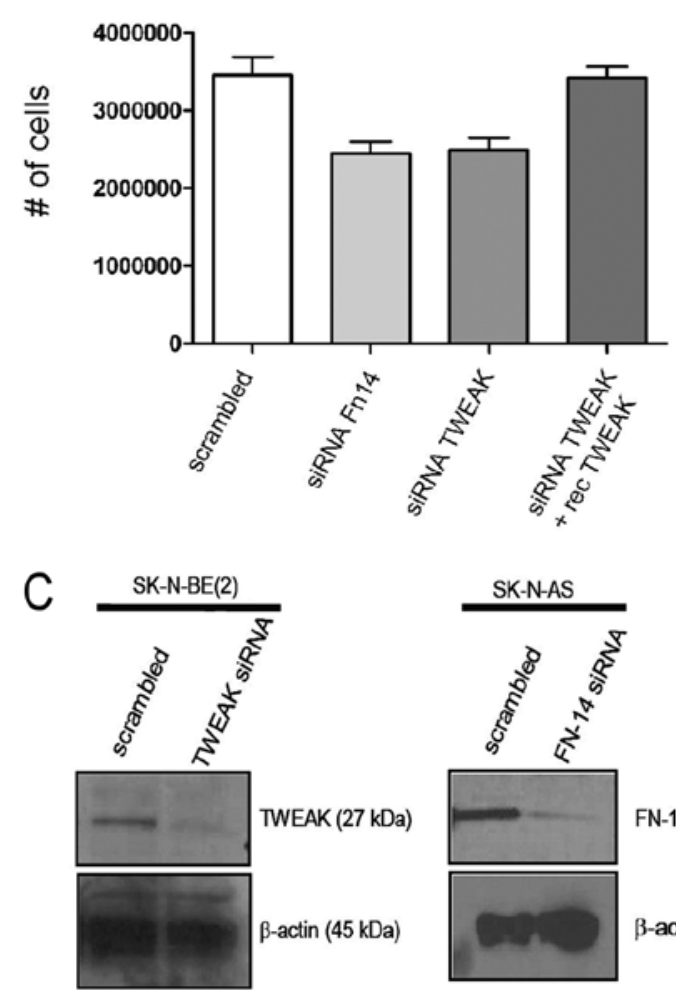

B

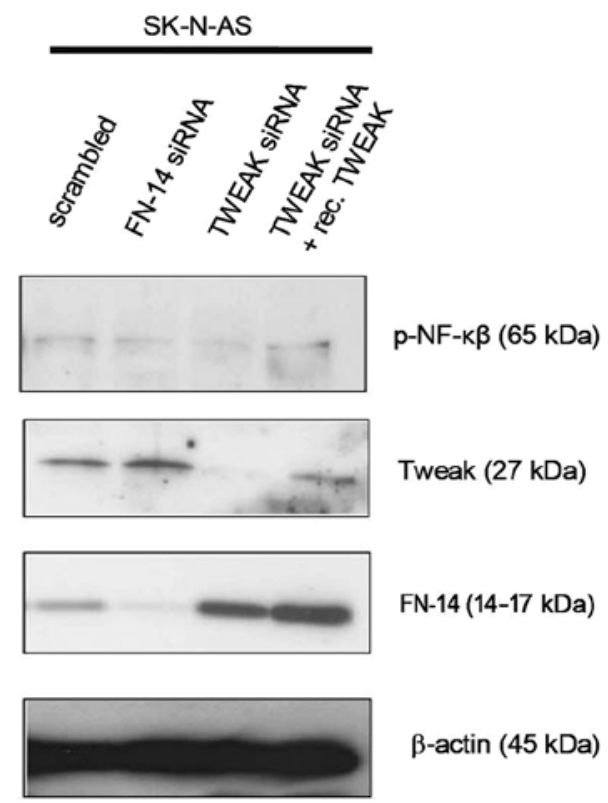

Figure 4. Silencing of TWEAK and Fn14 in neuroblastoma cells. (A) TWEAK and Fn14 expression are important for neuroblastoma growth. Trypan blue exclusion assay performed $72 \mathrm{~h}$ after initial transfection showing cell viability upon transfection of SK-N-AS cells with siRNA towards TWEAK or Fn14 in addition to siRNA transfection followed by recombinant TWEAK treatment (p<0.05). (B) Expression of TWEAK and Fn14 in SK-N-AS cells after transfection with scramble siRNA and siRNA towards TWEAK or Fn14. (C) Cell viability assay results were verified by western blot analysis showing a decrease in the endogenous protein expression of TWEAK in SK-N-BE(2) cells and Fn14 in SK-N-AS cells upon silencing with siRNA.
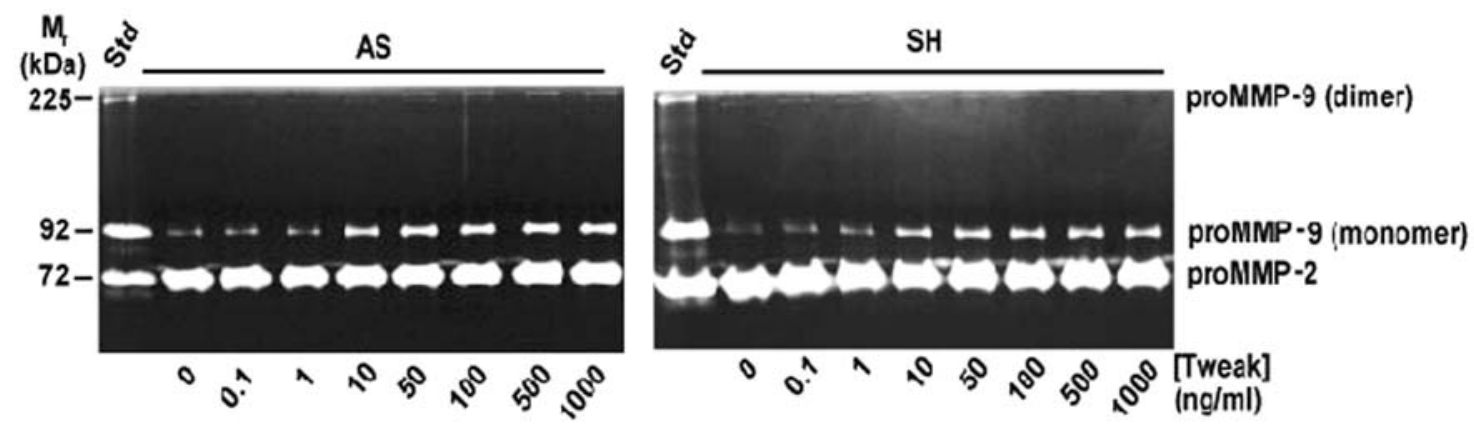

Figure 5. TWEAK induces MMP-9 activity in neuroblastoma cells. Gelatin zymography of TWEAK-treated SK-N-AS and SK-N-SH culture supernatants shows that TWEAK induces pro-MMP-9 (monomer) in a dose-dependent manner. MMP-2 production is constitutive.

of the ligand resulting in lowered receptor internalization and degradation of receptor-ligand complex. Additional western blotting was performed to also confirm the specific downregulation of TWEAK or Fn14 expression in SK-N-AS cells following siRNA transfection (Fig. 4C).

TWEAK induces MMP-9 expression in neuroblastoma cells. Since MMP-9 is a NF- $\kappa \mathrm{B}$ responsive gene and TWEAK has been shown to induce MMP-9 protein level in other cell systems $(21,22)$, we investigated the effect of TWEAK on the secretion of MMP-2 and MMP-9 in four different neuroblastoma cell lines. Zymography performed on conditioned medium from neuroblastoma cell lines revealed that MMP-2 was constitutively expressed, whereas TWEAK induced the release of MMP-9 in a dose-dependent manner in SK-N-AS and SK-N-SH cells (Fig. 5). Similar results were obtained for SK-N-BE(2) cells whereas SK-N-SY cells did not release MMP-9 upon stimulation with TWEAK (data not shown), indicating a heterogeneity with respect to TWEAK induction of MMP-9 in neuroblastoma cells. 


\section{Discussion}

The TNF superfamily of proteins has been implicated in the regulation of cell survival and proliferation. We have previously studied the role of tumor necrosis factor-related apoptosis inducing ligand (TRAIL) in neuroblastoma (37). In the present study, we describe the expression of TWEAK in neuroblastoma. TWEAK is a member of the TNF family of cytokines that acts on responsive cells via binding to a cell surface receptor called Fn14. TWEAK is a multifunctional cytokine expressed in a variety of normal tissue, but its expression has also been implicated in certain types of cancer [reviewed in refs. 16,18]. Equivalent to TWEAK, Fn14 is expressed in most cells and tissues (16) although the expression level is normally low. However, Fn14 expression can be induced by several cytokines, hormones and inflammatory mediators (16). A high expression of Fn14 has been detected in various adult cancers $(7,15,38)$ and Fn14 mRNA expression correlates with glioma grade and patient outcome (15). In addition, increased Fn14 mRNA and protein have been shown to be associated with disease progression in esophageal adenocarcinoma $(17,39)$ and cancer of the mammary (21).

We analyzed neuroblastoma primary tumors from different biological subsets and clinical stages, and detected TWEAK and Fn14 in all samples investigated (Table I and Fig. 1E). TWEAK and Fn14 mRNA and protein were all detected in various degrees in all neuroblastoma cell lines investigated (Fig. 1A and B), and soluble TWEAK was detected in supernatants from neuroblastoma cells (Fig. 1C). The discrepancy between mRNA and protein levels of TWEAK and Fn14 may have root in several aspects such as mRNA stability and posttranslational modification. Furthermore, the mRNA analysis was performed by reversal transcription PCR which is not a quantitative method. Immunofluorescence images of SK-N-AS cells demonstrate that the endogenously produced ligand and its receptor are co-localized to the cytoplasm, and to a certain degree in the nucleus (Fig. 1D). Interestingly, a 'short' variant of endogenously formed TWEAK possessing a nuclear localization sequence has been shown to colocalize with GSK3 $\beta$ in the nucleus of human neuroblastoma cells (40).

The significance of TWEAK and Fn14 expression in neuronal cells is not well understood. TWEAK has been shown to be expressed in primary murine neurons (41) and in the peripheral nervous system. TWEAK and Fn14 have been reported to regulate neurite outgrowth and regeneration (42), and a recent study has also demonstrated the role of TWEAK/ Fn14 in neurite extension in neural progenitor cells (11).

We observed an up-regulation of both TWEAK and Fn14 mRNA in several primary tumors from three publicly available European neuroblastoma data sets. It is presently unknown why the Fn14 gene is elevated in certain solid tumors. Fn14 gene amplification may possibly occur during the development and progression of these tumors although this is not frequently detected in neuroblastomas (43). Alternatively, the expression of Fn14 might be driven by a range of cytokines and growth factors produced within the tumor microenvironment. Additionally, TWEAK produced by tumor cells or other cells within the tumor microenvironment may activate Fn14 and NF- $\mathrm{KB}$ in cancer cells by use of a positive feedback loop (15). In this study, both TWEAK and Fn14 mRNA were found at significantly higher levels in a sub-set of neuroblastoma samples. Among 10 cases showing up-regulation of TWEAK (fold change >2), 8 also show up-regulation of Fn14 (fold change $>2$ ) supporting this hypothesis (Fig. 2).

To examine the effect of TWEAK on the proliferation of neuroblastoma cells, we stimulated serum-starved cells with an increasing concentration of TWEAK (1-1000 $\mathrm{ng} / \mathrm{ml})$ for 24 and $48 \mathrm{~h}$. In contrast to other reports using other types of tumor cells $(5,7,23)$, we did not detect any significant increased proliferation of neuroblastoma cells upon stimulation with TWEAK (data not shown). However, this is in line with results from studies on murine postnatal neural progenitor cells (11) and human embryonic kidney (HEK 293) cells (44). TWEAK stimulation through Fn14 activates the NF- $\mathrm{KB}$ signaling pathway in various cells and induces the expression of proinflammatory molecules $(16,45)$. Inflammatory mediators are critical components of tumor growth and the possibility that TWEAK produced by tumor cells may act on stromal cells within the tumor is supported by studies showing that TWEAK can induce secretion of cytokines from endothelial cells (7), fibroblasts (46) and macrophages (22). In addition, the activation of stromal cells may promote the infiltration of innate immune system cells that in turn may be potential sources for TWEAK and other pro-inflammatory cytokines. In our study, pro-inflammatory cytokines were shown to enhance TWEAK secretion by neuroblastoma cells (Fig. 1C). Therefore, inflammatory mediators within the tumor microenvironment may contribute to the further induction of TWEAK secretion by neuroblastoma cells.

Numerous genes have been described that are regulated by $\mathrm{NF}-\kappa \mathrm{B}$ and mediate the survival of cancer cells. These include genes such as MMP-9 (47), VEGF (48), and COX-2 (49), which have been closely associated with invasion and angiogenesis.

In the present study, we detected the nuclear expression of phospho-NF- $\mathrm{kB}$ in all primary neuroblastoma tissue samples by the use of immunohistochemistry (Fig. 1E). The phosphorylation of NF-кB (p65) was induced upon TWEAK stimulation of neuroblastoma cells as shown by western blot analysis (Fig. 3B). Furthermore, we demonstrate the translocation of NF-KB into the nucleus of TWEAK-stimulated neuroblastoma cells (Fig. 3A). A recent study demonstrated the the Fn14 expression in gastric cancer was inversely correlated with patient survival. Furthermore, expression level of Fn14 was shown to affect cell growth which in turn was mediated by NF- $\kappa \mathrm{B}$ activity (50).

Among proteases involved in tumor invasion and metastasis are the matrix metalloproteases (MMPs), a large family of endopeptidases which together can process all extracellular matrix proteins as well as other non-matrix proteins such as growth factors, cytokines and receptors $(51,52)$. Neuroblastoma cells are able to produce extracellular matrix degrading enzymes such as MMP-2 and MMP-9 (53-55). TWEAK has been shown to upregulate MMP-9 expression in both normal (22) and transformed cells $(21,56)$. Stimulating neuroblastoma cells with TWEAK induced the expression of MMP-9 in a dose-dependent manner as observed in gelatin zymograph. Neither of the cell lines responded to TWEAK stimulation by a change in MMP-2 expression (Fig. 5). These results suggest that TWEAK may have important functions during the metastasis conversion in neuroblastoma. 
In conclusion, our data demonstrate that TWEAK and Fn14 are expressed in neuroblastoma, and may play an important role in pro-survival features of the tumor. Given that targeted antibody therapy for Fn14 in several human xenografts results in significant antitumor effects $(57,58)$ a further evaluation of the mechanisms behind TWEAK and Fn14 expression may reveal new therapeutic options for neuroblastoma.

\section{Acknowledgements}

We wish to thank Anja Inkeri Vepså, Eli Berg and Kenneth Larsen for their technical assistance. This study was supported by grants from the Norwegian Cancer Society (DNK), The Ragnvarda F. Sörvik and Hakon Starheims Foundation, Norway, The Familien Blix Fond, The Swedish Children Cancer Foundation, The Swedish Cancer Society, Marta and Gunnar V Philipson Foundation, The Mary Bevé Foundation, Dammen Foundation, Karolinska Institutet and the Swedish Research Council.

\section{References}

1. Locksley RM, Killeen N and Lenardo MJ: The TNF and TNF receptor superfamilies: integrating mammalian biology. Cell 104: 487-501, 2001.

2. Bodmer JL, Schneider P and Tschopp J: The molecular architecture of the TNF superfamily. Trends Biochem Sci 27: 19-26, 2002.

3. Chicheportiche Y, Bourdon PR, Xu H, et al: TWEAK, a new secreted ligand in the tumor necrosis factor family that weakly induces apoptosis. J Biol Chem 272: 32401-32410, 1997.

4. Tran NL, McDonough WS, Savitch BA, Sawyer TF, Winkles JA and Berens ME: The tumor necrosis factor-like weak inducer of apoptosis (TWEAK)-fibroblast growth factor-inducible 14 (Fn14) signaling system regulates glioma cell survival via NFkappaB pathway activation and BCL-XL/BCL-W expression. J Biol Chem 280: 3483-3492, 2005.

5. Lynch CN, Wang YC, Lund JK, Chen YW, Leal JA and Wiley SR: TWEAK induces angiogenesis and proliferation of endothelial cells. J Biol Chem 274: 8455-8459, 1999.

6. Harada N, Nakayama M, Nakano H, Fukuchi Y, Yagita H and Okumura K: Pro-inflammatory effect of TWEAK/Fn14 interaction on human umbilical vein endothelial cells. Biochem Biophys Res Commun 299: 488-493, 2002.

7. Kawakita T, Shiraki K, Yamanaka Y, et al: Functional expression of TWEAK in human hepatocellular carcinoma: possible implication in cell proliferation and tumor angiogenesis. Biochem Biophys Res Commun 318: 726-733, 2004.

8. Donohue PJ, Richards CM, Brown SA, et al: TWEAK is an endothelial cell growth and chemotactic factor that also potentiates FGF-2 and VEGF-A mitogenic activity. Arterioscler Thromb Vasc Biol 23: 594-600, 2003 .

9. Tran NL, McDonough WS, Donohue PJ, et al: The human Fn14 receptor gene is up-regulated in migrating glioma cells in vitro and overexpressed in advanced glial tumors. Am J Pathol 162: 1313-1321, 2003.

10. Perper SJ, Browning B, Burkly LC, et al: TWEAK is a novel arthritogenic mediator. J Immunol 177: 2610-2620, 2006.

11. Hamill CA, Michaelson JS, Hahm K, Burkly LC and Kessler A: Age-dependent effects of TWEAK/Fn14 receptor activation on neural progenitor cells. J Neurosci Res 85: 3535-3544, 2007.

12. Marsters SA, Sheridan JP, Pitti RM, Brush J, Goddard A and Ashkenazi A: Identification of a ligand for the death-domaincontaining receptor Apo3. Curr Biol 8: 525-528, 1998.

13. Nakayama M, Kayagaki N, Yamaguchi N, Okumura K and Yagita H: Involvement of TWEAK in interferon gammastimulated monocyte cytotoxicity. J Exp Med 192: 1373-1380, 2000.

14. Meighan-Mantha RL, Hsu DK, Guo Y, et al: The mitogeninducible Fn14 gene encodes a type I transmembrane protein that modulates fibroblast adhesion and migration. J Biol Chem 274: 33166-33176, 1999.
15. Tran NL, McDonough WS, Savitch BA, et al: Increased fibroblast growth factor-inducible 14 expression levels promote glioma cell invasion via Rac1 and nuclear factor-kappaB and correlate with poor patient outcome. Cancer Res 66: 9535-9542, 2006.

16. Winkles JA: The TWEAK-Fn14 cytokine-receptor axis: discovery, biology and therapeutic targeting. Nat Rev Drug Discov 7: 411-425, 2008.

17. Watts GS, Tran NL, Berens ME, et al: Identification of Fn14/ TWEAK receptor as a potential therapeutic target in esophageal adenocarcinoma. Int J Cancer 121: 2132-2139, 2007.

18. Winkles JA, Tran NL, Brown SA, Stains N, Cunliffe HE and Berens ME: Role of TWEAK and Fn14 in tumor biology. Front Biosci 12: 2761-2771, 2007.

19. Winkles JA, Tran NL and Berens ME: TWEAK and Fn14: new molecular targets for cancer therapy? Cancer Lett 235: 11-17, 2006.

20. Li H, Mittal A, Paul PK, et al: Tumor necrosis factor-related weak inducer of apoptosis augments matrix metalloproteinase 9 (MMP-9) production in skeletal muscle through the activation of nuclear factor-kappaB-inducing kinase and p38 mitogenactivated protein kinase: a potential role of MMP-9 in myopathy. J Biol Chem 284: 4439-4450, 2009.

21. Michaelson JS, Cho S, Browning B, et al: Tweak induces mammary epithelial branching morphogenesis. Oncogene 24: 2613-2624, 2005.

22. Kim SH, Kang YJ, Kim WJ, et al: TWEAK can induce proinflammatory cytokines and matrix metalloproteinase-9 in macrophages. Circ J 68: 396-399, 2004.

23. Jakubowski A, Ambrose C, Parr M, et al: TWEAK induces liver progenitor cell proliferation. J Clin Invest 115: 2330-2340, 2005.

24. Maris JM, Hogarty MD, Bagatell R and Cohn SL: Neuroblastoma. Lancet 369: 2106-2120, 2007

25. De Preter K, Vandesompele J, Heimann P, et al: Human fetal neuroblast and neuroblastoma transcriptome analysis confirms neuroblast origin and highlights neuroblastoma candidate genes. Genome Biol 7: R84, 2006.

26. McArdle L,McDermott M, Purcell R, et al: Oligonucleotide microarray analysis of gene expression in neuroblastoma displaying loss of chromosome 11q. Carcinogenesis 25: 1599-1609, 2004.

27. Wilzen A, Nilsson S, Sjoberg RM, Kogner P, Martinsson T and Abel F: The Phox 2 pathway is differentially expressed in neuroblastoma tumors, but no mutations were found in the candidate tumor suppressor gene PHOX2A. Int J Oncol 34: 697-705, 2009.

28. Molenaar JJ, Ebus ME, Koster J, et al: Cyclin D1 and CDK4 activity contribute to the undifferentiated phenotype in neuroblastoma. Cancer Res 68: 2599-2609, 2008.

29. Brodeur GM, Pritchard J, Berthold F, et al: Revisions of the international criteria for neuroblastoma diagnosis, staging, and response to treatment. J Clin Oncol 11: 1466-1477, 1993

30. Sontag E, Sontag JM and Garcia A: Protein phosphatase $2 \mathrm{~A}$ is a critical regulator of protein kinase $\mathrm{C}$ zeta signaling targeted by SV40 small $t$ to promote cell growth and NF-kappaB activation. EMBO J 16: 5662-5671, 1997.

31. Johannessen M, Olsen PA, Sorensen R, Johansen B, Seternes OM and Moens U: A role of the TATA box and the general co-activator hTAF(II)130/135 in promoter-specific trans-activation by simian virus 40 small $t$ antigen. J Gen Virol 84: 1887-1897, 2003.

32. Malla N, Berg E, Uhlin-Hansen L and Winberg JO: Interaction of pro-matrix metalloproteinase-9/proteoglycan heteromer with gelatin and collagen. J Biol Chem 283: 13652-13665, 2008.

33. Winberg JO, Kolset SO, Berg E and Uhlin-Hansen L: Macrophages secrete matrix metalloproteinase 9 covalently linked to the core protein of chondroitin sulphate proteoglycans. J Mol Biol 304: 669-680, 2000.

34. Dogra C, Changotra H, Mohan S and Kumar A: Tumor necrosis factor-like weak inducer of apoptosis inhibits skeletal myogenesis through sustained activation of nuclear factor-kappaB and degradation of MyoD protein. J Biol Chem 281: 10327-10336, 2006.

35. Han S, Yoon K, Lee K, et al: TNF-related weak inducer of apoptosis receptor, a TNF receptor superfamily member, activates NF-kappa B through TNF receptor-associated factors. Biochem Biophys Res Commun 305: 789-796, 2003.

36. Saitoh T, Nakayama M, Nakano H, Yagita H, Yamamoto N and Yamaoka S: TWEAK induces NF-kappaB2 p100 processing and long lasting NF-kappaB activation. J Biol Chem 278: 36005-36012, 2003 
37. Johnsen JI, Pettersen I, Ponthan F, Sveinbjornsson B, Flaegstad T and Kogner P: Synergistic induction of apoptosis in neuroblastoma cells using a combination of cytostatic drugs with interferon-gamma and TRAIL. Int J Oncol 25: 1849-1857, 2004.

38. Feng SL, Guo Y, Factor VM, et al: The Fn14 immediate-early response gene is induced during liver regeneration and highly expressed in both human and murine hepatocellular carcinomas Am J Pathol 156: 1253-1261, 2000.

39. Wang S, Zhan M, Yin J, et al: Transcriptional profiling suggests that Barrett's metaplasia is an early intermediate stage in esophageal adenocarcinogenesis. Oncogene 25: 3346-3356, 2006.

40. De Ketelaere A, Vermeulen L, Vialard J, et al: Involvement of GSK-3beta in TWEAK-mediated NF-kappaB activation. FEBS Lett 566: 60-64, 2004.

41. Yepes M, Brown SA, Moore EG, Smith EP, Lawrence DA and Winkles JA: A soluble Fn14-Fc decoy receptor reduces infarct volume in a murine model of cerebral ischemia. Am J Pathol 166 511-520, 2005.

42. Tanabe K, Bonilla I, Winkles JA and Strittmatter SM: Fibroblast growth factor-inducible-14 is induced in axotomized neurons and promotes neurite outgrowth. J Neurosci 23: 9675-9686, 2003.

43. Caren H, Kryh H, Nethander M, et al: High-risk neuroblastoma tumors with 11q-deletion display a poor prognostic, chromosome instability phenotype with later onset. Proc Natl Acad Sci USA 107: 4323-4328, 2010.

44. Ho DH, Vu H, Brown SA, Donohue PJ, Hanscom HN and Winkles JA: Soluble tumor necrosis factor-like weak inducer of apoptosis overexpression in HEK293 cells promotes tumor growth and angiogenesis in athymic nude mice. Cancer Res 64 : 8968-8972, 2004.

45. Gao HX, Campbell SR, Burkly LC, et al: TNF-like weak inducer of apoptosis (TWEAK) induces inflammatory and proliferative effects in human kidney cells. Cytokine 46: 24-35, 2009.

46. Chicheportiche Y, Chicheportiche R, Sizing I, et al: Proinflammatory activity of TWEAK on human dermal fibroblasts and synoviocytes: blocking and enhancing effects of anti-TWEAK monoclonal antibodies. Arthritis Res 4: 126-133, 2002.

47. Yokoo T and Kitamura M: Antioxidant PDTC induces stromelysin expression in mesangial cells via a tyrosine kinase-AP-1 pathway. Am J Physiol 270: F806-F811, 1996.

48. Huang S, Robinson JB, Deguzman A, Bucana CD and Fidler IJ: Blockade of nuclear factor-kappaB signaling inhibits angiogenesis and tumorigenicity of human ovarian cancer cells by suppressing expression of vascular endothelial growth factor and interleukin 8. Cancer Res 60: 5334-5339, 2000.
49. Schmedtje JF Jr, Ji YS, Liu WL, DuBois RN and Runge MS: Hypoxia induces cyclooxygenase-2 via the NF-kappaB p65 transcription factor in human vascular endothelial cells. J Biol Chem 272: 601-608, 1997.

50. Kwon OH, Park SJ, Kang TW, et al: Elevated fibroblast growth factor-inducible 14 expression promotes gastric cancer growth via nuclear factor-kappaB and is associated with poor patient outcome. Cancer Lett 314: 73-81, 2012.

51. Tonti GA, Mannello F, Cacci E and Biagioni S: Neural stem cells at the crossroads: MMPs may tell the way. Int J Dev Biol 53: 1-17, 2009.

52. Hadler-Olsen E, Fadnes B, Sylte I, Uhlin-Hansen L and Winberg JO: Regulation of matrix metalloproteinase activity in health and disease. FEBS J 278: 28-45, 2011

53. Farina AR, Tiberio A, Tacconelli A, Cappabianca L, Gulino A and Mackay AR: Identification of plasminogen in Matrigel and its activation by reconstitution of this basement membrane extract. Biotechniques 21: 904-909, 1996.

54. Ara T, Fukuzawa M, Kusafuka T, et al: Immunohistochemical expression of MMP-2, MMP-9, and TIMP-2 in neuroblastoma: association with tumor progression and clinical outcome. J Pediatr Surg 33: 1272-1278, 1998.

55. Ribatti D, Surico G, Vacca A, et al: Angiogenesis extent and expression of matrix metalloproteinase- 2 and -9 correlate with progression in human neuroblastoma. Life Sci 68: 1161-1168, 2001.

56. Huang M, Narita S, Tsuchiya N, et al: Overexpression of Fn14 promotes androgen-independent prostate cancer progression through MMP-9 and correlates with poor treatment outcome. Carcinogenesis 32: 1589-1596, 2011.

57. Culp PA, Choi D, Zhang Y, et al: Antibodies to TWEAK receptor inhibit human tumor growth through dual mechanisms. Clin Cancer Res 16: 497-508, 2010.

58. Michaelson JS, Kelly R, Yang L, Zhang X, Wortham K and JosepH IB: The anti-Fn14 antibody BIIB036 inhibits tumor growth in xenografts and patient derived primary tumor models and enhances efficacy of chemotherapeutic agents in multiple xenograft models. Cancer Biol Ther 13: 812-821, 2012. 